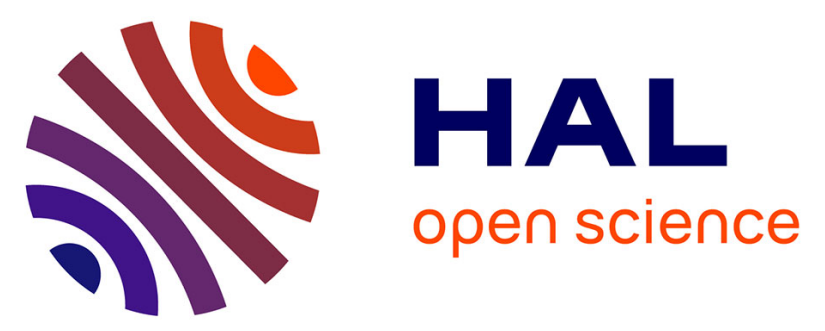

\title{
Circulating phospholipid profiling identifies portal contribution to NASH signature in obesity
}

Kavya Anjani, Marie Lhomme, Nataliya Sokolovska, Christine Poitou, Judith Aron-Wisnewsky, Jean-Luc Bouillot, Philippe Lesnik, Pierre Bedossa, Anatol Kontush, Karine Clement, et al.

\section{To cite this version:}

Kavya Anjani, Marie Lhomme, Nataliya Sokolovska, Christine Poitou, Judith Aron-Wisnewsky, et al.. Circulating phospholipid profiling identifies portal contribution to NASH signature in obesity. Journal of Hepatology, 2014, 62 (4), pp.905-912. 10.1016/j.jhep.2014.11.002 hal-01090208

\section{HAL Id: hal-01090208 \\ https://hal.sorbonne-universite.fr/hal-01090208}

Submitted on 3 Dec 2014

HAL is a multi-disciplinary open access archive for the deposit and dissemination of scientific research documents, whether they are published or not. The documents may come from teaching and research institutions in France or abroad, or from public or private research centers.
L'archive ouverte pluridisciplinaire HAL, est destinée au dépôt et à la diffusion de documents scientifiques de niveau recherche, publiés ou non, émanant des établissements d'enseignement et de recherche français ou étrangers, des laboratoires publics ou privés. 
Circulating phospholipid profiling identifies portal contribution to NASH signature in obesity

Kavya Anjani ${ }^{1,2,3}$, Marie Lhomme ${ }^{3}$, Nataliya Sokolovska ${ }^{1,2,3}$, Christine Poitou ${ }^{1,2,3}$, Judith AronWisnewsky ${ }^{1,2,3}$, Jean-Luc Bouillot ${ }^{5}$, Philippe Lesnik ${ }^{3,4,6}$, Pierre Bedossa ${ }^{7}$, Anatol Kontush ${ }^{3,4,6}$, Karine Clement ${ }^{1,2,3}$, Isabelle Dugail ${ }^{1,2,3} *$ and Joan Tordjman ${ }^{1,2,3} *$

* contributed equally to this work.

1. Sorbonne Universités, UPMC Université Paris 06, UMR_S 1166 I, ICAN, Nutriomics team, F-75005, Paris, France;

2. INSERM, UMR_S U1166, Nutriomics team, F-75013, Paris, France;

3. Institute of Cardiometabolism and Nutrition, ICAN, AP-HP, Pitié-Salpêtrière Hospital, F75013, Paris, France;

4. INSERM, UMR_S U1166, Dyslipidemia, Inflammation and atherosclerosis team, F75013, Paris, France;

5. Assistance Publique-Hôpitaux de Paris, Ambroise Pare Hospital, Surgery Department, Boulogne-Billancourt, France;

6. Sorbonne Universités, UPMC Université Paris 06, UMR_S 1166 I, ICAN, Dyslipidemia, Inflammation and atherosclerosis team, F-75005, Paris, France;

7. Assistance Publique-Hôpitaux de Paris, Beaujon Hospital, Pathology Department, Clichy, France; Centre de Recherche Bichat-Beaujon, INSERM U773, University Paris-Diderot, Paris, France; 


\section{Corresponding authors:}

Joan Tordjman and Isabelle Dugail

Centre de Recherche des Cordeliers, UMR S 1166, Team 6, 91 bd de l'Hôpital, 75013 Paris, France. Tel: +33144278075 Fax: +33144278076

Email: joan.tordjman@crc.jussieu.fr; isabelle.dugail@inserm.fr

Abstract word count: 250

Manuscript word count: $\mathbf{5 0 3 0}$

Number of figures: 4

Number of tables: 1

Abbreviations: NAFLD, non alcoholic fatty liver disease; NASH, nonalcoholic steatohepatitis; LC-MS, liquid chromatography coupled mass spectrometry; PL, glycerophospholipids; SL, sphingolipids; PC, glycerophosphatidylcholines; LPC, lyso-glycerophosphatidylcholines; PE, glycerophosphatidylethanolamines; PI, glycerophosphatidylinositols; $\quad$ PG, glycerophosphatidylglycerols; PS, glycerophosphatidylserines; PA, glycerophosphatidicacids; SM, sphingomyelins; Cer, ceramides; ALT, alanine aminotransferase; AST, aspartate aminotransferase; GGT, gamma-glutamyl transpeptidase; IL-6, interleukin 6; hsCRP, high 
sensitive C-reactive protein; SAF, steatosis, activity and fibrosis; BMI, body mass index; HbA1c, glycated hemoglobin; FCS, fat cell size

\section{Conflict of interest: None}

\section{Financial support:}

We thank Assistance Publique - Hôpitaux de Paris (APHP) and the Direction of Clinical Research, the Programme Hospitalier de Recherche Clinique for supporting clinical investigations (AOR 02076 to K.C.). This work was also supported by the European Commission (Collaborative Project FP6 "Hepatic and adipose tissue and functions in the metabolic syndrome', HEPADIP, see http://www.hepadip.org/, contract LSHM-CT-2005018734). The research leading to these results received funding from the European Union Seventh Framework Program (FP7/2007-2013) under grant agreement no. Health-F2-2009241762, for the FLIP project. We thank the Société Française de Nutrition (SFN) for financial support (JAW).

\section{Authors' contributions:}

KA performed the experiments and data analysis, drafted the manuscript, and prepared figures.

ML performed LC-MS/MS analysis, NS performed machine learning analysis and prepared figures. CP, JAW and JLB provided clinical samples, PL and AK participated in study design and manuscript editing. KC, ID and JT conceived the study, participated in its design, coordination, and data analysis, and helped write and edit the manuscript. All authors read, participated in editing the manuscript, and approved the final manuscript. 


\begin{abstract}
Background \& Aims: Nonalcoholic steatohepatitis (NASH) is characterized by steatosis, lobular inflammation, hepatocyte ballooning with fibrosis in severe cases and high prevalence in obesity. We aimed at defining NASH signature in morbid obesity by mass spectrometry-based lipidomic analysis. Methods: Systemic blood before and 12 months post bariatric surgery along with portal blood and adipose tissue lipid efflux collected at the time of surgery from obese women were analyzed (9 structural classes, 150 species). Results: Increased concentrations of several Glycerophosphocholines (PC), Glycerophosphoethanolamines (PE),
\end{abstract} Glycerophosphoinositols (PI), Glycerophosphoglycerols (PG), Lyso-Glycerophosphocholines (LPC), and Ceramides (Cer) were detected in systemic circulation of NASH subjects. Weight loss post-surgery (12 months) improved the levels of liver enzymes, as well as several lipids, but most PG and Cer species remained elevated. Analysis of lipids from hepatic portal system at the time of surgery revealed limited lipid alterations compared to systemic circulation, but PG and PE classes were found significantly increased in NASH subjects. We evaluated the contribution of visceral adipose tissue to lipid alterations in portal circulation by measuring adipose tissue lipid efflux ex vivo, which demonstrated only minor alterations in NASH subjects. Interestingly, integration of clinical and lipidomic data (portal and systemic) led us to define a NASH signature in which lipids and clinical parameters are equal contributors. Conclusion: Circulatory (portal and systemic) phospholipid profiling and clinical data defines NASH signature in morbid obesity. We report weak contribution of visceral adipose tissue to NASH-related portal lipid alterations, suggesting possible contribution from other organs draining into hepatic portal system.

Keywords: Lipidomics, Visceral Adipose tissue, Glycerophosphatidylglycerols, Glycerophosphatidylethanolamines, bariatric surgery 


\section{Introduction}

Non-alcoholic fatty liver disease (NAFLD) comprises steatosis to nonalcoholic steatohepatitis (NASH). Insulin resistance and obesity are some of the major risk factors for NAFLD [1] along with age [2], gender [3], ethnicity [4][5] and genetic variants such as patatin-like phospholipase domain-containing 3 (PNPLA3) [6]. Incidence of NAFLD in the general population is estimated in the range of $6-14 \%$ whereas in specific population such as in morbid obesity it is reported to be as high as $84-96 \%$, comprising $25-55 \%$ with NASH and $34-47 \%$ with liver fibrosis [7]. NAFLD is associated with elevated cardiovascular risk [8][9][10] with increased fat mass as an important mediator. We showed that accumulation of inflammatory cells in visceral and not subcutaneous adipose tissue is associated with severity of NAFLD in morbid obesity [11][12]. Extent of hepatic inflammation and fibrosis also associated with visceral fat independent of insulin resistance and hepatic steatosis [13][14][15]. These findings indicate potential contribution of visceral fat to worsening liver condition through release of bioactive molecules such as cytokines into hepatic portal system draining into liver, thus supporting the portal hypothesis. Increased liver load with molecules including free fatty acids (FFA) [16][17] and inflammatory mediators [18] from visceral fat were shown to contribute to altered hepatic function [19][20][21][22][23].

Lipids are obvious players in NAFLD. Mass spectrometry based lipidomic approaches offer detailed analysis of disease-associated changes in lipid profiles. Early studies reported perturbations in hepatic lipid classes during progression from normal to fatty liver and NASH, accumulation of triacylglycerols (TAG), diacylglycerols (DAG), free cholesterols, elevated Lyso-Glycerophosphocholines (LPC) and a shift towards more saturated fatty acids [24]. Gorden 
et al. [25] also observed increased DAG in steatotic compared to normal liver, but reported mostly unchanged; Glycerophosphocholines (PC), Glycerophosphoethanolamines (PE) and Glycerophosphoinositols (PI). Despite a potential for pathophysiological and non-invasive diagnostic information, analysis of circulatory lipidome in NAFLD is limited to date. Puri et al. found decreased plasma essential polyunsaturated fatty acids (PUFAs), increased (MUFAs) and lipoxygenase metabolites in NASH subjects [26]. Recently, variation in circulatory TAG profiles served as a basis to define phenotypic heterogeneity in NASH i.e. PNPLA3 or obesity-associated NASH [27].

Though comprehensive analysis of all lipids still remain a challenge, we focused on Phospholipids (PL) and Sphingolipids (SL), which represent nearly half of the plasma lipidome in humans [28]. We performed MS-based lipidomic analysis on systemic and hepatic portal circulation simultaneously to characterize obese NASH subjects and examine portal lipid input to liver. Contribution of visceral adipose tissue to NASH was also investigated, as well as improvement in NASH associated lipid alterations following gastric bypass-induced weight loss. Advanced statistical machine learning method was used for integration of lipidomic and clinical data to define a NASH signature in morbid obesity, visualized as a Bayesian network.

\section{Patients and methods}

\section{Study cohort and sample collection}

Forty-six morbidly obese women, candidates for gastric bypass surgery were recruited at PitiéSalpêtrière Hospital (ICAN, Institute of Cardiometabolism and Nutrition). All subjects gave written informed consent. Local ethics committee approved the present clinical investigations. 
Exclusion criteria include autoimmune, inflammatory or infectious diseases, viral hepatitis, cancer, known alcohol consumption ( $>20$ g/day) or kidney diseases. Among patients with NASH, 19 were diabetics and treated with: Metformin $(n=13)$, Insulin $(n=1)$, Sulfamide $(n=1)$, incretinomimetic drug (i.e. GLP1 analogs, n=2) and 2 without treatment. Sera were collected from systemic blood following an over-night fast. Blood from right epiploic vein connected to portal trunk, therefore termed "portal blood" was collected during surgery. Biopsies of omental, subcutaneous and mesenteric adipose tissues and liver were obtained from all subjects during surgery and stored at $-80^{\circ} \mathrm{C}$ or fixed until further analyses. Clinical characteristics of the cohort are summarized in Table $\mathbf{1 .}$

\section{Biochemical analyses}

Serum glucose, triglycerides, total and HDL-cholesterol, alanine aminotransferase (ALT), aspartate aminotransferase (AST), gamma-glutamyl transpeptidase (GGT) and fibrinogen were measured. Serum insulin concentrations were determined by Bi-INSULIN IRMA (CisBio International, Gif-sur-Yvette, France), leptin and adiponectin were assayed by radioimmunoassay (Linco Research, Saint Louis, MI, USA), interleukin-6 (IL-6) was measured by ELISA (QuantikineUS, R\&D System Europe Ltd, Abingdon, UK), high-sensitive C-reactive protein (hsCRP) was measured using an IMMAGE automatic immunoassay system (BeckmanCoulter, Fullerton, CA, USA).

\section{Liver histopathology}

The liver biopsy was formalin-fixed, paraffin-embedded and serial sections were stained. Minimal staining included Haematoxylin and Eosin, PicroSirius Red and Perls. All biopsies were 
reviewed by a single liver pathologist and graded on SAF score, absence or presence of NASH was evaluated according to diagnostic algorithm for NASH [29]. No NASH subjects had no histological signs of steatosis, lobular and portal inflammation and fibrosis.

\section{Lipidomics}

Lipidomic measurements were based on the method by Camont et al. [30]. Briefly, lipids were extracted by cold acidified methanol: chloroform with internal standard for each lipid class. All standards were purchased from Avanti Polar Lipids (Alabaster, AL, USA). Eight PL subclasses; PC, LPC, PE, PI, PG, PA, Lyso-glycerophosphoethanolamines (LPE), Glycerophosphoserines (PS) and two SL subclasses Sphingomyelins (SM) and Ceramides (Cer) comprising 158 species were assayed and quantified by LC/MS/MS (Shimadzu, Kyoto, Japan, and AB Sciex, Framingham, MA, USA).

\section{Bayesian network}

We employed computational biology for combined analysis of clinical and lipidomic data. Supervised machine learning using sqsvm (Squared Hindge Loss Classification) algorithm from gglasso package in $\mathrm{R}$ environment was used. Optimal computational model based on minimal 10-cross folds validation error rate was chosen. Interdependencies between clinical and lipidomic data was revealed by learning a Bayesian network using the $\mathrm{R}$ package bnlearn, where nodes represent variables of interest and edges between the nodes represent relationships between them. The resulting network was visualized using Cytoscape, open source software.

\section{Statistical analysis}


All statistical analyses were performed in R (http://www.r-project.org/). Data are expressed as mean \pm SEM. In most analysis, significant differences between groups were estimated using unpaired Wilcoxon rank sum test while paired Wilcoxon rank sum test was performed to compare same group at different time points. For age adjustment, we used linear models after $\underline{\log \text { transformation of the variables and have used the function } \operatorname{lm}() \text { for fitting linear models of } \mathrm{R}}$ package "stats" (R version 3.0.1 ). A p-value of less than 0.05 was considered to be statistically significant.

\section{Results}

\section{Characterization of morbidly obese women with NASH}

Morbidly obese women (BMI matched) were grouped as NASH or no NASH based on histological assessment of liver biopsies. NASH subjects were significantly older than no NASH with $68 \%$ being type 2 diabetic with higher fasting glucose and HbA1c. NASH subjects had higher levels of AST, ALT, GGT, fasting triglycerides, serum IL-6 and lower fat mass and adiponectin (Table 1).

\section{Change in circulatory phospholipid profile in obese women with NASH}

Mass spectrometry-based approach was used to profile PL and SL species in serum obtained from patients' systemic blood under fasting condition. A total of 158 lipid species were identified and quantified from PC, LPC, PE, LPE, PI, PS, PG, PA, SM and Cer. Lipidomic data projected onto the plane depicting maximum inertia (total variance) of the dataset i.e., the first two principal components explain $45 \%$ of variance in the data and was able to distinguish between NASH and no NASH subjects (Fig. 1A). Type 2 diabetic and non-diabetic subjects discriminated to a lesser extent (supp Fig. 1). Significant NASH-associated lipid changes were 
visualized as heat map presenting ratio of each lipid concentration in individual NASH subject vs. mean of no NASH subjects, and revealed a large number of altered lipids in NASH subjects (Fig. 1B). Sum of concentrations of individual lipid species within same class showed significant increase in PC, PE, PI, PG and Cer (Fig. 1C). After adjustment for age, which differed between the two groups, PI and Cer were not found altered. LPC was not found elevated in the NASH group but positively correlated with age and negatively with obesity duration (Suppl Fig 2). A characteristic feature of circulating lipidome is the wide concentration range over three log amplitude between different lipid classes. For example, most abundant PC and SM circulate within a 50-100 mg/dL range, whereas PE and LPC are found at 10 fold lower concentration, followed by PI, Cer (1-2 mg/dL) and PS, PG, PA with concentrations below $0.1 \mathrm{mg} / \mathrm{dL}$ (Fig. 1C). Individual species within a class also vary in concentration range, those bearing the common C16 and C18 fatty acids being the most abundant. Therefore, to evaluate the contribution of individual lipid species we plotted their relative abundance against their ratio NASH/no NASH concentration. This revealed that NASH associates with modifications of not only high but also of several low abundant species (Fig. 1D).

Gastric bypass results in partial reversal of phospholipid alteration in obese women with NASH

Gastric bypass surgery-induced weight loss associated with improvements in BMI, fat mass, fasting glucose, insulin, HbA1c, triglycerides, HDL-C and hsCRP (Table 1). NASH subjects showed significant decrease in serum levels of liver enzymes 12 months post surgery that reached values similar to those of no NASH subjects, suggesting amelioration of liver condition (Fig. 2A). Despite this improvement, we cannot conclude on liver rescue in this population as there is no biopsy proven diagnosis of NASH post surgery. Therefore, we retained the initial 
grouping of subjects based on their baseline biopsy-proven diagnosis for lipid profiling. Post surgery lipidomic data indicate that several lipids, mainly PG and Cer, remain significantly elevated in systemic circulation of NASH subjects (Fig. 2B and C) in addition to a few intermediate abundant PC and PE (Fig. 2D). Overall lipid profile before and after surgery visualized as volcano plots of log mean ratio $\mathrm{NASH} /$ no $\mathrm{NASH}$ against p-value (ratio over 0 indicates increase in NASH), shows much fewer alteration after surgery. Twelve months following gastric bypass surgery was associated with lower number of affected lipids in NASH subjects compared to baseline (Fig. 2E). Detailed analysis showed that PI was mostly resolved while PC and PE were partially resolved but PG and Cer remained elevated in NASH subjects 12 months after gastric bypass surgery (Fig. 2F).

\section{Partial contribution of adipose tissue depots to NASH-associated phospholipid alterations}

We investigated potential contribution of adipose tissue to NASH-associated alterations in lipid profiles by estimating ex vivo lipid efflux. For this purpose calibrated omental, mesenteric and subcutaneous adipose tissue biopsies from NASH and no NASH subjects obtained at the time of surgery were incubated for 24 hours in media containing BSA as a lipid acceptor. PL and SL efflux into the medium was quantified and individual lipids were expressed as mol\%. Interestingly, we were able to detect all PL (identified in serum) under these conditions of ex vivo adipose tissue efflux. Compared to serum, the distribution of lipids effluxed from three adipose tissue depots mainly differed in LPC: PC ratio and higher efflux of PG and PA (Supp Fig. 3A). Comparison of profiles of adipose tissue lipid efflux from NASH and no NASH subjects showed very few alterations. Only few species, not overlapping in the three adipose tissue depots were less efficiently effluxed from adipose tissue depots of NASH subjects (Supp 
Fig. 3B). Remarkably, PG (36:1) from omental and subcutaneous and PG (34:1) from omental adipose tissue were among the lipids effluxed at significantly higher rate from adipose tissue of NASH subjects (Supp Fig. 3C). Overall, these data suggest weak contribution of adipose tissue towards circulating PL alterations in obese women with NASH.

Lipid alterations in the hepatic portal system and association with liver pathology in morbid obesity

We next investigated lipid profiles from the hepatic portal system, known to transport molecules from visceral organs to liver. Portal blood was collected from right epiploic vein during gastric bypass surgery and plasma was used for lipidomic analysis. Compared to systemic circulation, fewer alterations were observed in portal phospholipidome of obese women with NASH (Fig. 3A vs. Fig. 2E baseline). Visualization of heat map indicates lipid alterations in PC, PE, PI and PG in hepatic portal circulation of NASH subjects (Fig. 3B) with major alterations in PE and PG classes (Fig. 3C). The most abundant PE(36:2) along with 6 other low abundant species and 2 high and 2 low abundant PG species were significantly increased in NASH subjects (supplementary Fig. 4). As a lipid class, PC and PI did not appear altered (Fig. 3C) even if few species such as $\mathrm{PC}(36: 1,38: 5,40: 4,40: 5)$ and $\mathrm{PI}(32: 0,32: 1,34: 2,36: 4,40: 4$, 40:5) were significantly increased in NASH (supplementary Fig. 4). Cer that was altered systemically was unaltered in the portal circulation of NASH subjects (Fig. 1B and 3B). Low abundant species predominantly from PG and PE and few PC and PI dominated NASH associated lipid alteration in hepatic portal circulation (Fig 3D). 


\section{NASH signature in morbid obesity}

Since bioclinical and lipidomic data contribute to NASH characterization, we learnt a Bayesian network to identify the most relevant associations with NASH. The network was constructed from baseline values from 15 bioclinical variables including mesenteric and omental adipocyte size and 61 and 24 lipidomic variables from systemic and portal circulation respectively. The nodes of the network were pre-selected by a sparse support vector machine algorithm. Directly interacting nodes with NASH, considered Markov blanket of NASH were bioclinical variables; age, fasting glycemia, serum adiponectin and fibrinogen, tissue phenotypes; mesenteric and omental fat cell size (FCS) and lipid species; systemic PC(40:4), portal pPC(40:4), PI(40:5) and portal pCer(d18:2/14:0). These variables thus influenced or were influenced directly by NASH. Width of lines in the network are proportional to the weight of dependencies and suggest that relationships between NASH and PC(40:4) (systemic and portal) and omental FCS are stronger than with other nodes. The influence of age on NASH status also appeared as an important factor. The Bayesian network also revealed strong dependencies between the portal lipids pPC(40:4), pSM(42:3) and pCer(d18:2/14:0) and between systemic PG(34:1) and PI(40:5). As expected fasting glycemia, directly linked to NASH has a strong interaction with $\mathrm{HbAlc}$ and ALT (Fig. 4A). Altogether these data highlight that circulating (systemic and also portal blood) phospholipids interact with clinical and tissue parameters to define NASH signature in morbid obesity. Because portal blood lipids is not available in routine patient investigation, we tested for added value of lipid profiling systemic blood only in NASH prediction. Using variables selected from machine learning algorithms, ROC curves indicated that selected lipids alone had a poor predictive value over clinical variables, but combination of selected lipids and biological parameters slightly improved NASH prediction (Fig 4B). 


\section{Discussion}

Liver disease is commonly associated with obesity, but not all obese subjects develop NASH. This study explored morbidly obese subjects with or without liver alterations in a combined analysis of not only systemic but also portal lipids. A main outcome is that, in this context of morbid obesity with patients stratified for NASH, the composition of PL in the portal circulation differs, indicating selective alterations in lipid input to the liver. Nevertheless, fewer lipid species are impacted in portal circulation compared to massive changes that dominate systemic blood, reported in this and previous studies [26].

Several circulating protein markers have previously been defined in NASH and associated insulin-resistance such as cytokeratin 18 or Fetuin A [31][32]. Focusing on lipids, we identified 4 PL significantly contributing to the NASH signature. Therefore, these lipids represent putative candidates in NASH-associated liver deterioration. In particular, we have identified NASHrelated changes in several prominent PG species indicating that phosphatidylglycerols might have physiopathological relevance to NASH. The integration of lipidomic and clinical variables to construct a Bayesian network identified 15 most significant contributors and also revealed their interdependencies. Among those, there were 7 lipid species (4 from portal and 3 from systemic circulation) and 8 clinical features. This approach demonstrates the interest in combining usual clinical variables and large-scale measurements to identify complex NASH signatures and potentially provides new basis for hypothesis in NASH development in morbid obesity. 
We confirmed the importance of fasting glycemia, HbAlc and adiponectinemia, linked to glucose homeostasis and NASH [33][34]. Insulin resistance is common feature in NASH [35][36]. Nevertheless, in the present study fasting insulin levels are equally elevated in NASH and no NASH subjects. This may be due to specific features, particularly the high proportion of NASH patients with diabetes $(68 \%)$ in our cohort, especially the high proportion of patient receiving anti-diabetic insulin treatment (and mainly metformin). This particularity, in addition to the well-known insulin-resistance of morbidly obese patients might explain why LPCs, a lipid class associated with insulin sensitivity, does not show up in our study. Despite normalization of liver enzymes after gastric bypass surgery [37], we showed that weight loss can only partially attenuate NASH-related alterations in systemic PL profiles at least one year after surgery. Persistence of some lipid alterations after surgery could be linked to unresolved glycemic status (only 4 among 17 subjects resolved their type 2 diabetes following gastric bypass surgery). It remains to explore these patients after longer-term follow-up to examine whether the targeted lipid species further change or stabilize in parallel to the evolution in clinical parameters. The older age of NASH subjects could also affect liver outcome but we could not find age-matched subjects in the two clinical groups. However adjustment for age of systemic lipid concentrations confirms significant PL alterations (i.e.PE, PG, PC) in NASH patients.

Regarding lipid alterations, the Bayesian network revealed a surprisingly high dependency of NASH to portal PC(40:4), a low abundant species with potential role as a signaling molecule that may warrant further investigation. Also, $\mathrm{SM}(42: 3)$ and a short chain (C14) Ceramide are the only SL with intriguingly decreased concentrations in portal circulation of NASH subjects and showed strong links with NASH but the biological significance is yet to be evaluated.

Most interestingly, about half of lipid species associated with NASH showed altered concentrations in the portal circulation. We initially hypothesized that in obesity, both omental 
and mesenteric fat tissues drained by portal blood could be determinant NASH contributors by releasing lipids. However our results may rather highlight the importance of other visceral organs, than visceral fat. Indeed our ex vivo observations revealed limited changes in adipose tissue lipid efflux from NASH and no NASH subjects. Moreover, in the Bayesian network, none of the lipid nodes showed strong dependencies with omental fat cell size, whereas this tissue phenotype per se appeared as a strong contributor in NASH signature. This suggests that omental fat involvement is through fatty acid sequestration rather than release of specific lipid species. On the other, association of adipose fat cell size with NASH in the network could reflect increased portal release of inflammatory mediators by hypertrophied fat cells that need to be further investigated. In addition, dysregulated hepatokines could also participate as reported recently by Stefan et al. [38].

PE and PG are the two major lipid classes elevated in portal circulation of NASH subjects, and two species (36:2 and 34:1) with high abundance among PG both contribute to NASH signature. Remarkably, PG and PE are mostly represented in bacterial membranes as opposed to eukaryotic cells predominantly composed of PC. Besides a role for gut microbiota dysbiosis in obesity [39] and the importance of microbial diversity in individual responses of obese subjects to nutritional intervention [40], changes in gut microbiome of children with or without NASH [41] have been reported. Therefore it is possible that elevated portal PG and PE in NASH could be linked to altered gut microbiota and, represent circulating microbial products possibly derived from bacterial translocation. Since PE and PG are elevated not only in portal but also in systemic circulation of obese NASH subjects, we propose the concept of gut microbiota-related hyperphospholipidemia as a potential new actor in NAFLD.

In conclusion, we showed that specific PL alteration in hepatic portal circulation, also observed in systemic circulation is characteristic of NASH in morbid obesity. Since portal lipid 
deregulation is weakly contributed by visceral adipose tissue, this suggests a possible contribution from gut microbiome. 
Acknowledgments: We thank Dr. Florence Marchelli (Center for Research on Human Nutrition, Pitié-Salpêtriére Hospital, Paris, France), for patient recruitment, data collection, and sampling. We thank Patricia Bonjour for histochemistry. We greatly thank Omran Allatif for his help in data presentation. We thank Mrs. Nathalie Colnot for providing technical assistance for tissue processing. 


\section{References}

[1] Ruhl CE, Everhart JE. Determinants of the association of overweight with elevated serum alanine aminotransferase activity in the United States. Gastroenterology. 2003;124:71-9.

[2] Targher G, Bertolini L, Padovani R, Rodella S, Tessari R, Zenari L, et al. Prevalence of nonalcoholic fatty liver disease and its association with cardiovascular disease among type 2 diabetic patients. Diabetes Care. 2007;30:1212-8.

[3] Arun J, Clements RH, Lazenby AJ, Leeth RR, Abrams GA. The prevalence of nonalcoholic steatohepatitis is greater in morbidly obese men compared to women. Obes. Surg. 2006;16:1351-8.

[4] Mohanty SR, Troy TN, Huo D, O’Brien BL, Jensen DM, Hart J. Influence of ethnicity on histological differences in non-alcoholic fatty liver disease. J. Hepatol. 2009;50:797-804.

[5] Bambha K, Belt P, Abraham M, Wilson LA, Pabst M, Ferrell L, et al. Ethnicity and nonalcoholic fatty liver disease. Hepatology. 2012;55:769-80.

[6] Romeo S, Kozlitina J, Xing C, Pertsemlidis A, Cox D, Pennacchio LA, et al. Genetic variation in PNPLA3 confers susceptibility to nonalcoholic fatty liver disease. Nat. Genet. 2008;40:1461-5.

[7] Clark JM. The epidemiology of nonalcoholic fatty liver disease in adults. J. Clin. Gastroenterol. 2006;40 Suppl 1:S5-10.

[8] Bhatia LS, Curzen NP, Calder PC, Byrne CD. Non-alcoholic fatty liver disease: a new and important cardiovascular risk factor? Eur. Heart J. 2012;33:1190-200.

[9] Targher G, Mantovani A, Pichiri I, Rigolon R, Dauriz M, Zoppini G, et al. Non-alcoholic fatty liver disease is associated with an increased prevalence of atrial fibrillation in hospitalized patients with type 2 diabetes. Clin. Sci. (Lond). 2013;125:301-9.

[10] Verrijken A, Francque S, Mertens I, Prawitt J, Caron S, Hubens G, et al. Prothrombotic factors in histologically proven NAFLD and NASH. Hepatology. 2013;

[11] Tordjman J, Divoux A, Prifti E, Poitou C, Pelloux V, Hugol D, et al. Structural and inflammatory heterogeneity in subcutaneous adipose tissue: relation with liver histopathology in morbid obesity. J. Hepatol. 2012;56:1152-8.

[12] Cancello R, Tordjman J, Poitou C, Guilhem G, Bouillot JL, Hugol D, et al. Increased infiltration of macrophages in omental adipose tissue is associated with marked hepatic lesions in morbid human obesity. Diabetes. 2006;55:1554-61.

[13] Van der Poorten D, Milner K-L, Hui J, Hodge A, Trenell MI, Kench JG, et al. Visceral fat: a key mediator of steatohepatitis in metabolic liver disease. Hepatology. 2008;48:44957. 
[14] Tordjman J, Poitou C, Hugol D, Bouillot J-L, Basdevant A, Bedossa P, et al. Association between omental adipose tissue macrophages and liver histopathology in morbid obesity: influence of glycemic status. J. Hepatol. 2009;51:354-62.

[15] Eguchi Y, Mizuta T, Sumida Y, Ishibashi E, Kitajima Y, Isoda H, et al. The pathological role of visceral fat accumulation in steatosis, inflammation, and progression of nonalcoholic fatty liver disease. J. Gastroenterol. 2011;46 Suppl 1:70-8.

[16] Bjorntorp P. "Portal" adipose tissue as a generator of risk factors for cardiovascular disease and diabetes. Arterioscler. Thromb. Vasc. Biol. 1990;10:493-496.

[17] Bergman RN, Ader M. Free fatty acids and pathogenesis of type 2 diabetes mellitus. Trends Endocrinol. Metab. 2000;11:351-6.

[18] Rytka JM, Wueest S, Schoenle EJ, Konrad D. The Portal Theory Supported by Venous Drainage - Selective Fat Transplantation. 2011;60.

[19] Hotamisligil GS. Mechanisms of TNF-alpha-induced insulin resistance. Exp. Clin. Endocrinol. Diabetes. 1999;107:119-25.

[20] Steppan CM, Bailey ST, Bhat S, Brown EJ, Banerjee RR, Wright CM, et al. The hormone resistin links obesity to diabetes. Nature. 2001;409:307-12.

[21] Berg AH, Combs TP, Du X, Brownlee M, Scherer PE. The adipocyte-secreted protein Acrp30 enhances hepatic insulin action. Nat. Med. 2001;7:947-53.

[22] Kabir M, Catalano KJ, Ananthnarayan S, Kim SP, Van Citters GW, Dea MK, et al. Molecular evidence supporting the portal theory: a causative link between visceral adiposity and hepatic insulin resistance. Am. J. Physiol. Endocrinol. Metab. 2005;288:E454-61.

[23] Jensen MD. Role of body fat distribution and the metabolic complications of obesity. J. Clin. Endocrinol. Metab. 2008;93:S57-63.

[24] Puri P, Baillie R a, Wiest MM, Mirshahi F, Choudhury J, Cheung O, et al. A lipidomic analysis of nonalcoholic fatty liver disease. Hepatology. 2007;46:1081-90.

[25] Gorden DL, Ivanova PT, Myers DS, McIntyre JO, VanSaun MN, Wright JK, et al. Increased diacylglycerols characterize hepatic lipid changes in progression of human nonalcoholic fatty liver disease; comparison to a murine model. PLoS One. 2011;6:e22775.

[26] Puri P, Wiest MM, Cheung O, Mirshahi F, Sargeant C, Min H-K, et al. The plasma lipidomic signature of nonalcoholic steatohepatitis. Hepatology. 2009;50:1827-38.

[27] Hyysalo J, Gopalacharyulu P, Bian H, Hyötyläinen T, Leivonen M, Jaser N, et al. Circulating Triacylglycerol Signatures in Nonalcoholic Fatty Liver Disease Associated With the I148M Variant in PNPLA3 and With Obesity. Diabetes. 2014;63:312-22. 
[28] Quehenberger O, Armando AM, Brown AH, Milne SB, Myers DS, Merrill AH, et al. Lipidomics reveals a remarkable diversity of lipids in human plasma. J. Lipid Res. 2010;51:3299-305.

[29] Bedossa P, Poitou C, Veyrie N, Bouillot J-L, Basdevant A, Paradis V, et al. Histopathological algorithm and scoring system for evaluation of liver lesions in morbidly obese patients. Hepatology. 2012;56:1751-9.

[30] Camont L, Lhomme M, Rached F, Le Goff W, Nègre-Salvayre A, Salvayre R, et al. Small, dense high-density lipoprotein-3 particles are enriched in negatively charged phospholipids: relevance to cellular cholesterol efflux, antioxidative, antithrombotic, antiinflammatory, and antiapoptotic functionalities. Arterioscler. Thromb. Vasc. Biol. 2013;33:2715-23.

[31] Anty R, Iannelli A, Patouraux S, Bonnafous S, Lavallard VJ, Senni-Buratti M, et al. A new composite model including metabolic syndrome, alanine aminotransferase and cytokeratin-18 for the diagnosis of non-alcoholic steatohepatitis in morbidly obese patients. Aliment. Pharmacol. Ther. 2010;32:1315-22.

[32] Stefan N, Häring H-U. Circulating fetuin-A and free fatty acids interact to predict insulin resistance in humans. Nat. Med. 2013;19:394-5.

[33] Gupte P, Amarapurkar D, Agal S, Baijal R, Kulshrestha P, Pramanik S, et al. Nonalcoholic steatohepatitis in type 2 diabetes mellitus. J. Gastroenterol. Hepatol. 2004;19:854-8.

[34] Ma H, Xu C, Xu L, Yu C, Miao M, Li Y. Independent association of HbA1c and nonalcoholic fatty liver disease in an elderly Chinese population. BMC Gastroenterol. 2013;13:3.

[35] Musso G, Cassader M, De Michieli F, Rosina F, Orlandi F, Gambino R. Nonalcoholic steatohepatitis versus steatosis: adipose tissue insulin resistance and dysfunctional response to fat ingestion predict liver injury and altered glucose and lipoprotein metabolism. Hepatology. 2012;56:933-42.

[36] Boza C, Riquelme A, Ibañez L, Duarte I, Norero E, Viviani P, et al. Predictors of nonalcoholic steatohepatitis (NASH) in obese patients undergoing gastric bypass. Obes. Surg. 2005;15:1148-53.

[37] Mathurin P, Hollebecque A, Arnalsteen L, Buob D, Leteurtre E, Caiazzo R, et al. Prospective study of the long-term effects of bariatric surgery on liver injury in patients without advanced disease. Gastroenterology. 2009;137:532-40.

[38] Stefan N, Häring H-U. The role of hepatokines in metabolism. Nat. Rev. Endocrinol. 2013;9:144-52.

[39] Ley RE, Bäckhed F, Turnbaugh P, Lozupone CA, Knight RD, Gordon JI. Obesity alters gut microbial ecology. Proc. Natl. Acad. Sci. U. S. A. 2005;102:11070-5. 
[40] Cotillard A, Kennedy SP, Kong LC, Prifti E, Pons N, Le Chatelier E, et al. Dietary intervention impact on gut microbial gene richness. Nature. 2013;500:585-8.

[41] Zhu L, Baker SS, Gill C, Liu W, Alkhouri R, Baker RD, et al. Characterization of gut microbiomes in nonalcoholic steatohepatitis (NASH) patients: a connection between endogenous alcohol and NASH.

Hepatology. 2013;57:601-9. 
Table 1

\begin{tabular}{|c|c|c|c|c|}
\hline & \multicolumn{2}{|c|}{ No NASH $(n=24)$} & \multicolumn{2}{|l|}{ NASH $(n=22)$} \\
\hline & Baseline & 12 months & Baseline & 12 months \\
\hline Age (years) & $36.42 \pm 2.4$ & & $48.55 \pm 2.5^{\# \#}$ & \\
\hline BMI $\left(\mathrm{kg} / \mathbf{m}^{2}\right)$ & $46.93 \pm 1.0$ & $31.61 \pm 1.2 * * * *$ & $45.06 \pm 1.2$ & $33.75 \pm 1.3 * * * *$ \\
\hline Percent Fat mass (\%) & $50.32 \pm 0.7$ & $38.70 \pm 1.3 * * * *$ & $46.81 \pm 0.8^{\# \#}$ & $40.96 \pm 1.1 * * * *$ \\
\hline Fasting Insulin $(\mu \mathrm{IU} / \mathrm{ml})$ & $17.21 \pm 2.0$ & $8.18 \pm 1.1^{* *}$ & $25.91 \pm 3.6$ & $12.72 \pm 4.1 * *$ \\
\hline Fasting glucose (mM) & $4.93 \pm 0.1$ & $4.55 \pm 0.1 * * *$ & $6.88 \pm 0.4^{\# \# \# ~}$ & $5.63 \pm 0.3 * * * \$ \$$ \\
\hline HbA1c (\%) & $5.58 \pm 0.1$ & $5.25 \pm 0.1 * * * *$ & $6.91 \pm 0.3^{\# \# \#}$ & $5.84 \pm 0.2 * * * * \$ \$$ \\
\hline Triglycerides (mM) & $1.02 \pm 0.1$ & $0.83 \pm 0.1 * *$ & $1.89 \pm 0.2^{\# \# \#}$ & $1.24 \pm 0.1 * * \$$ \\
\hline Cholesterol (mM) & $4.67 \pm 0.2$ & $4.36 \pm 0.1$ & $4.68 \pm 0.2$ & $4.60 \pm 0.2$ \\
\hline HDL-C (mM) & $1.32 \pm 0.1$ & $1.62 \pm 0.1 * * *$ & $1.17 \pm 0.1$ & $1.54 \pm 0.1 * * *$ \\
\hline ApoA1 (mM) & $1.54 \pm 0.1$ & $1.56 \pm 0.1$ & $1.39 \pm 0.1$ & $1.66 \pm 0.1 *$ \\
\hline ApoB (mM) & \pm 0.03 & $0.75 \pm 0.03 * *$ & $0.94 \pm 0.1$ & $0.82 \pm 0.05$ \\
\hline ASAT (IU/L) & $21.46 \pm 0.9$ & $23.62 \pm 1.2$ & $32.91 \pm 3.1^{\# \#}$ & $25.20 \pm 1.3^{*}$ \\
\hline ALAT (IU/L) & $21.25 \pm 1.6$ & $23.46 \pm 2.3$ & $38.36 \pm 4.1^{\# \#}$ & $22.45 \pm 1.8 * *$ \\
\hline GGT (mg/dl) & $32.21 \pm 6.9$ & $25.75 \pm 7.4^{* *}$ & $47.91 \pm 5.8^{\# \#}$ & $17.95 \pm 2.1 * * * *$ \\
\hline Leptin (ng/ml) & $63.00 \pm 6.3$ & $23.81 \pm 3.9 * * * *$ & $47.29 \pm 3.8$ & $26.63 \pm 4.0 * * *$ \\
\hline Adiponectin $(\mu \mathrm{g} / \mathrm{ml})$ & $4.46 \pm 0.4$ & $8.95 \pm 0.8 * * * *$ & $3.48 \pm 0.4^{\#}$ & $5.98 \pm 0.6 * * *$ \\
\hline IL-6 (pg/ml) & $3.11 \pm 0.3$ & $4.19 \pm 1.6$ & $4.28 \pm 0.4^{\#}$ & $4.38 \pm 0.8$ \\
\hline hsCRP (mg/L) & $0.97 \pm 0.2$ & $0.17 \pm 0.03 * * * *$ & $1.06 \pm 0.2$ & $0.22 \pm 0.06^{* * *}$ \\
\hline Fibrinogen (g/l) & $4.58 \pm 0.2$ & $4.19 \pm 0.2$ & $3.80 \pm 0.2^{\#}$ & $4.16 \pm 0.2$ \\
\hline
\end{tabular}


\# no NASH vs. NASH at baseline (non parametric unpaired Wilcoxon test) in women candidate for bariatric surgery. ${ }^{\$}$ no NASH vs. NASH at 12 months post surgery (non parametric unpaired Wilcoxon test). * Baseline vs. 12 months post surgery (non parametric paired test Wilcoxon test) $* \mathrm{p}<0.05$; ** $\mathrm{p}<0.01$; *** $\mathrm{p}<0.001$; **** $\mathrm{p}<$ 0.0001

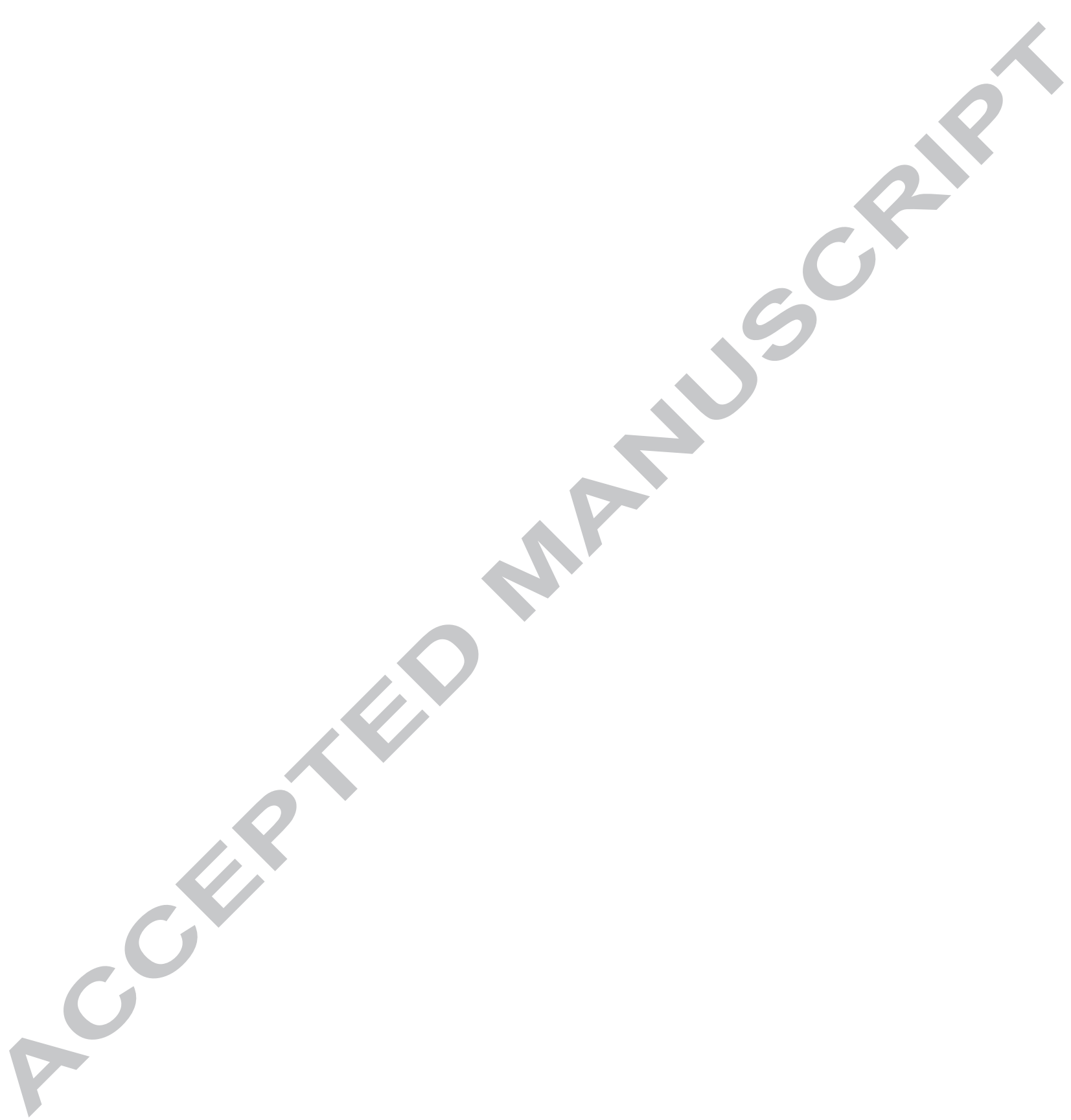




\section{Legends}

Fig. 1. NASH-associated alteration in serum lipids in morbidly obese women (A) Principal component analysis of systemic serum lipidomics data differentiate NASH and no NASH subjects. (B) Heat map of ratio [NASH/mean no-NASH] for significantly altered lipids. Rows indicate NASH subjects, columns represent distinct lipid species from PC, SM, PE, LPC, PI, Cer, PS, PG and PA. (C) Concentration of structurally related lipid classes in subjects with or without NASH. $* \mathrm{p}<0.05 * * \mathrm{p}<0.01$ (D) Lipid abundance relative to total phospholipids of significantly altered species (block circles) and unaltered species (crosses). Size of symbols is inversely proportional to $\mathrm{p}$-value,

Fig. 2. Gastric bypass surgery associated serum lipid alteration (A) AST, ALT and GGT levels before and 12 months post -surgery $* \mathrm{p}<0.05 * * \mathrm{p}<0.01 * * * \mathrm{p}<0.001$. (B) Heat map of significantly altered serum lipids 12 months post-surgery. (see legend Fig. 1. B). (C) Concentration of structurally-related lipid classes 12 months post surgery. $* \mathrm{p}<0.05$. (D) NASH-associated changes after surgery; relative abundance of lipids (see Fig. 1. D). (E) Phospholipidome alteration at baseline and 12 months post surgery. (F) Heat map of significant $\mathrm{NASH}$-associated lipid changes in serum at baseline (before surgery) and 12 months post surgery.

Fig. 3. NASH-associated lipid alteration in hepatic portal circulation (A) Volcano plot of NASH-associated lipid alterations in hepatic portal system (B) Heat map of significantly altered 
portal lipids (see Fig. 1. B) (C) Concentration of structurally related lipid classes $* \mathrm{p}<0.05 * * \mathrm{p}$ $<0.01$ (D) NASH-associated changes in portal circulation; relative abundance of lipids (see Fig. 1. D).

Fig. 4. NASH signature based on lipidomic and clinical data. A: Interdependencies among lipids (blue) from systemic and portal circulation( prefix "p"), clinical parameters (yellow), and NASH (red) constructed by Bayesian network. Width of the edges is proportional to the strength of dependencies. * denotes negative association with NASH. B: ROC curves for NASH using selected systemic lipids. ROC curves were generated using SVM selected variables for: 1) clinical parameters alone; 2) systemic lipids alone and 3) combined clinical and systemic lipids parameters. 
A

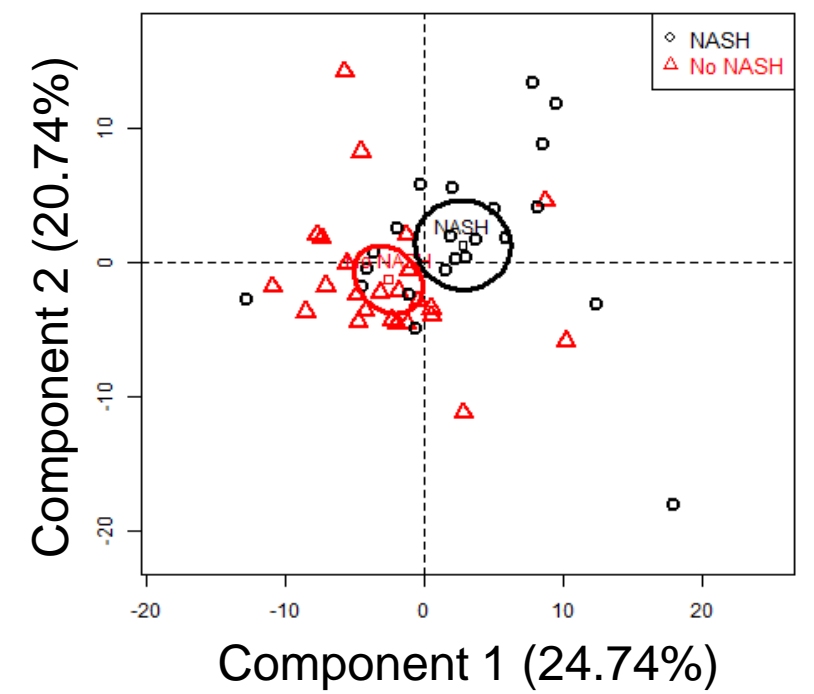

C

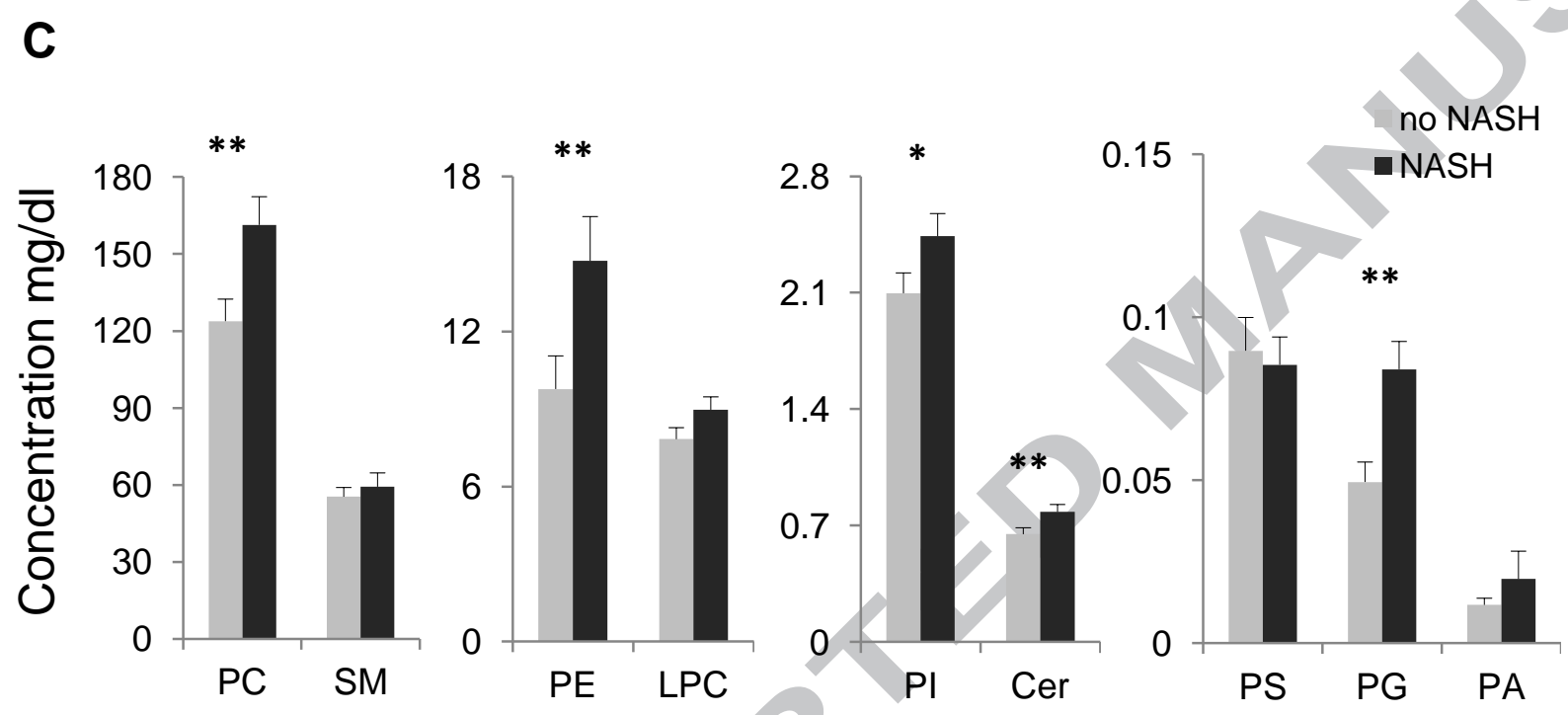

B EPTED MANIUSCRIPT

$-0.5$

0.5

Log [NASH/mean no NASH]
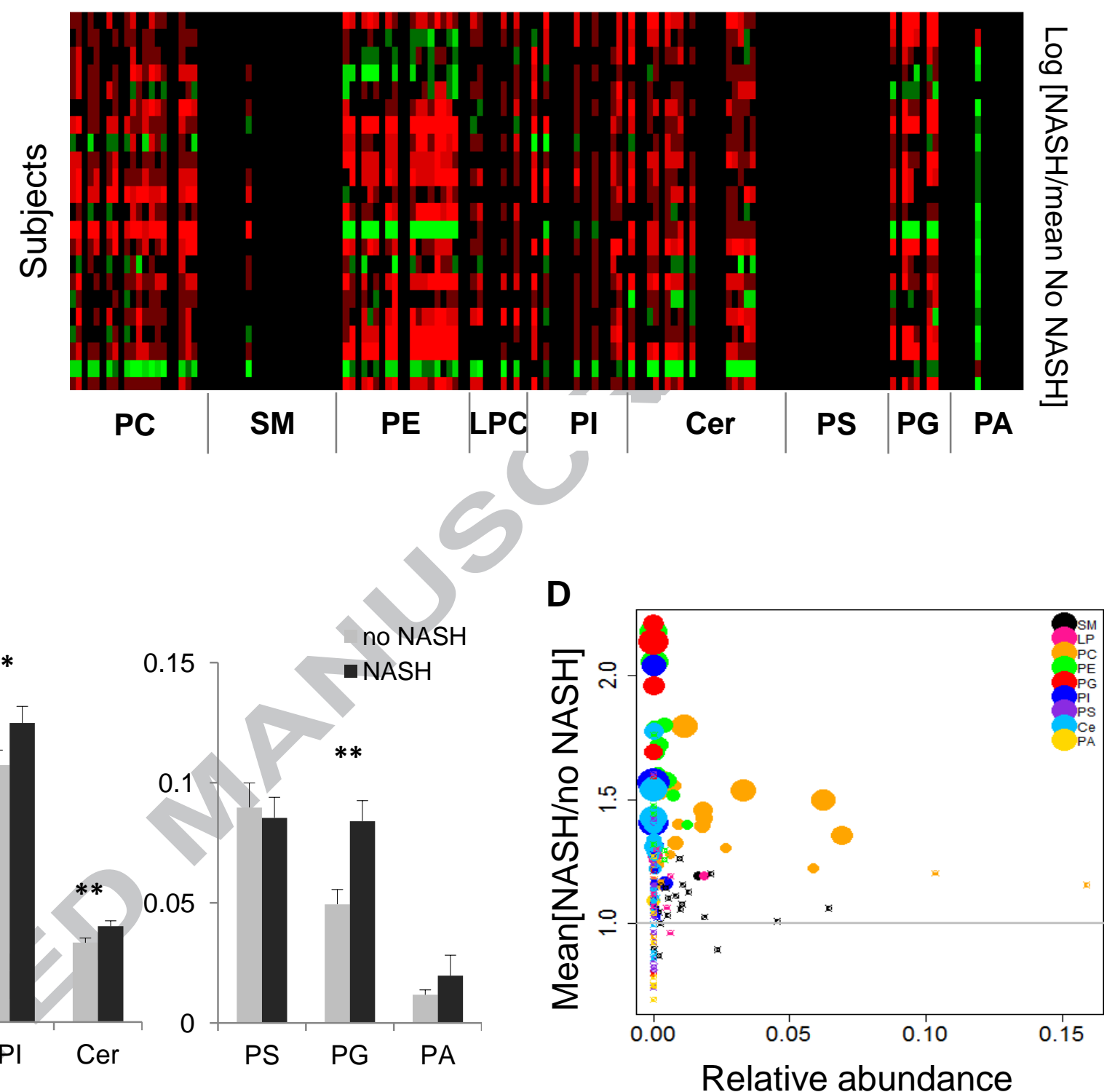

Figure 1 


\section{E}

\section{Systemic Baseline}
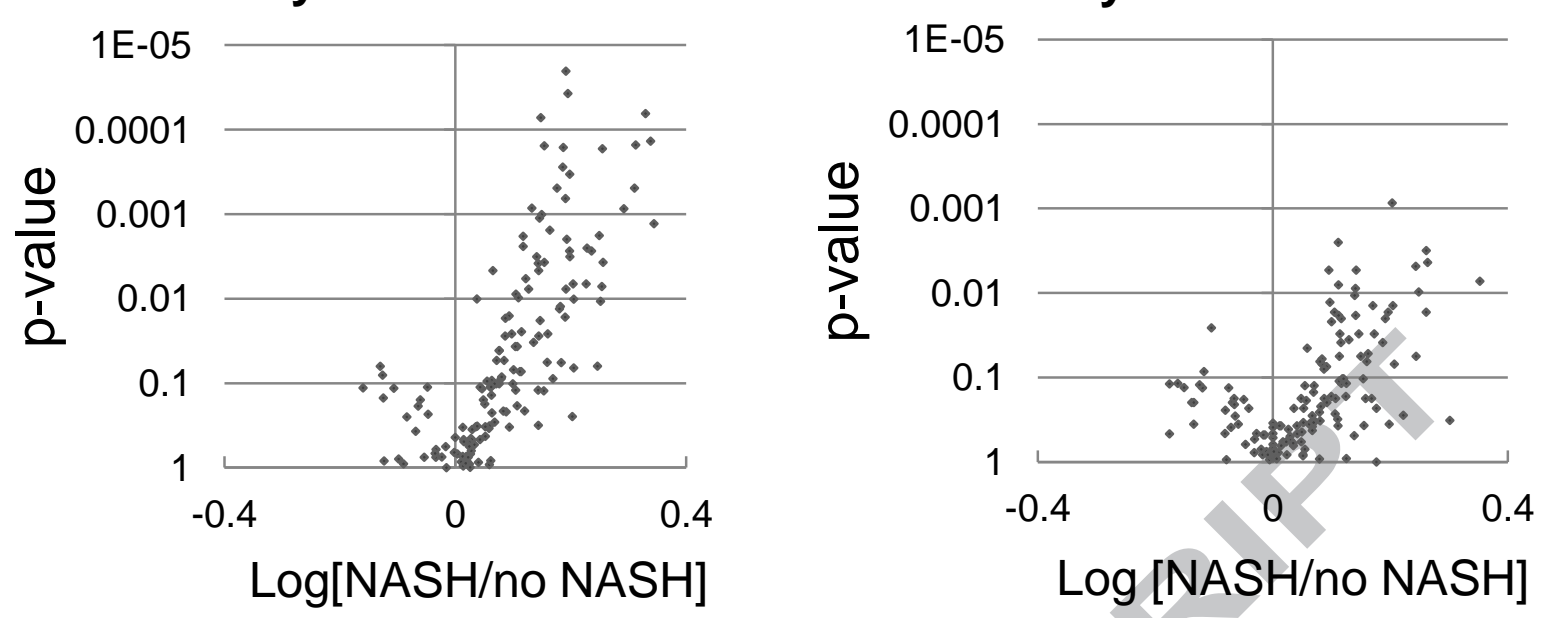

Figure 2
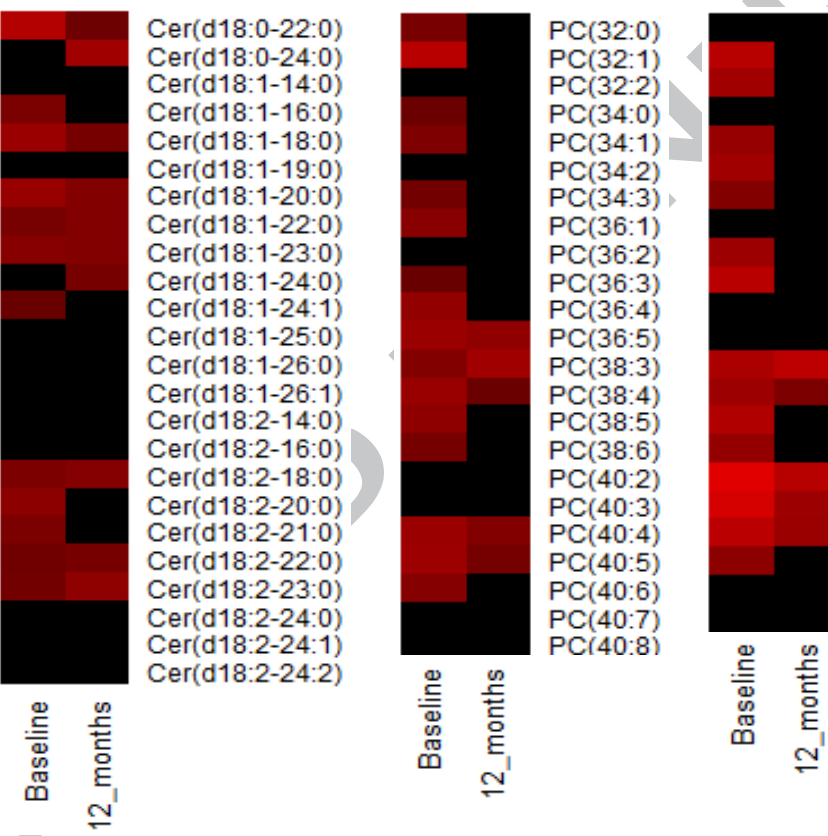

$P E(32: 1)$
$P E(34: 0)$
$P E(34: 1)$
$P E(34: 2)$
$P E(34: 3)$
$P E(36: 1)$
$P E(36: 2)$
$P E(36: 3)$
$P E(36: 4)$
$P E(36: 5)$
$P E(36: 6)$
$P E(38: 2)$
$P E(38: 3)$
$P E(38: 4)$
$P E(38: 5)$
$P E(38: 6)$
$P E(40: 4)$
$P E(40: 5)$
$P E(40: 6)$
$P E(40: 7)$
$P E(42: 6)$
$P E(42: 7)$

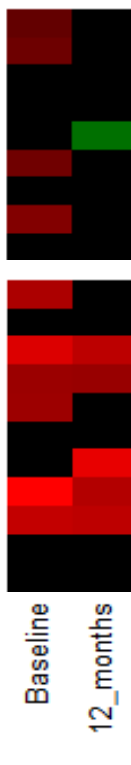

$\operatorname{LPC}(16: 0)$ $\operatorname{LPC}(16: 1)$

Cer(d18:0-24:0)

Cer(d18:1-14:0)

Cer(d18:1-16:0)

Cer(d18:1-19:0)

Cer(d18:1-20:0)

Cer(d18:1-22:0)

Cer(d18:1-23:0)

Cer(d18:1-24:0)

Cer(d18:1-26:0)

Cer(d18:1-26:1)

Cer(d18.2-18.0)

Cer(d18.2-20:0)

Cer(d18:2-21:0)

Cer(d18:2-22:0)

Cer(d18:2-23:0)

Cer(d18:2-24:0)

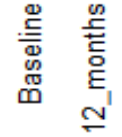

Cer(d18.2-24.2)

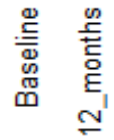

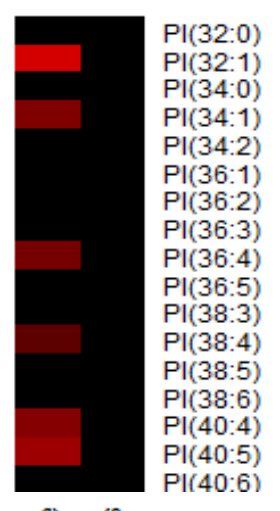

LPC (18:1)

LPC (18:2)

$\operatorname{LPC}(20: 3)$

$\operatorname{LPC}(20: 4)$

$\operatorname{LPC}(22: 5)$

$\mathrm{LPC}(22: 6)$

$P G(34: 1)$

$P G(34: 2)$

$P G(36: 1)$

PG(36:2)

$P G(36: 3)$

$P G(36: 4)$

PG(38:2)

$\mathrm{PG}(38 \cdot 3)$

$P G(38: 4)$

$\mathrm{PG}(38.5)$

PG(38:6)

Log [NASH/no NASH]

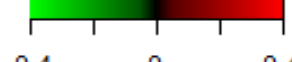




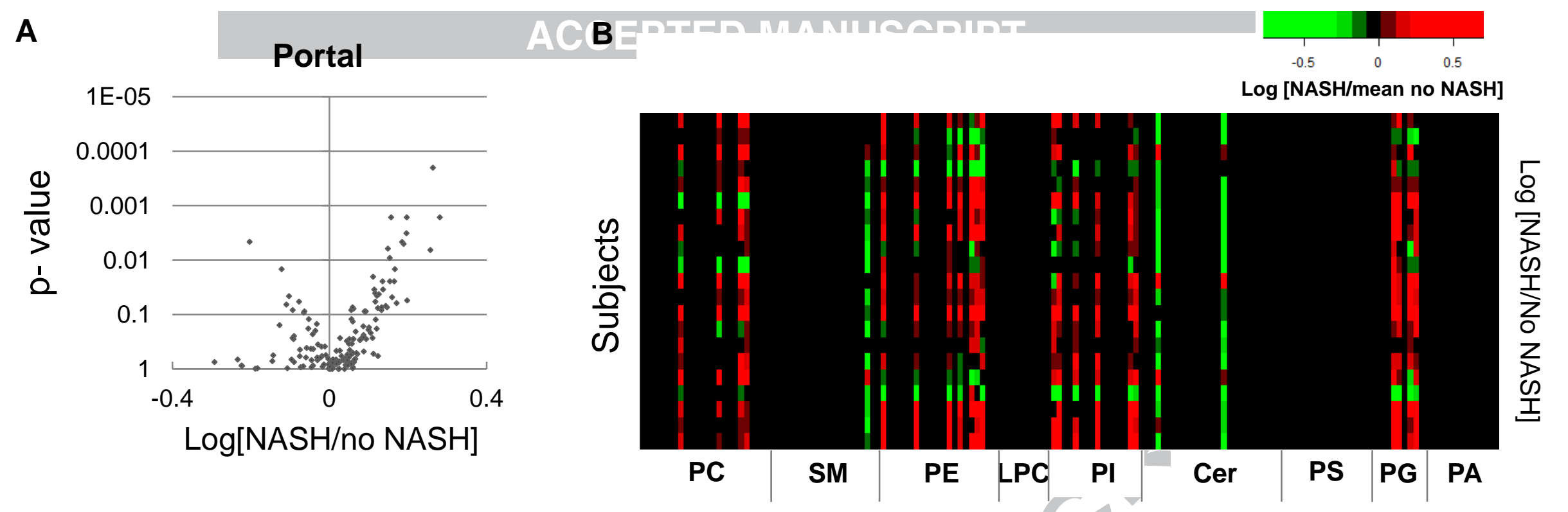

C

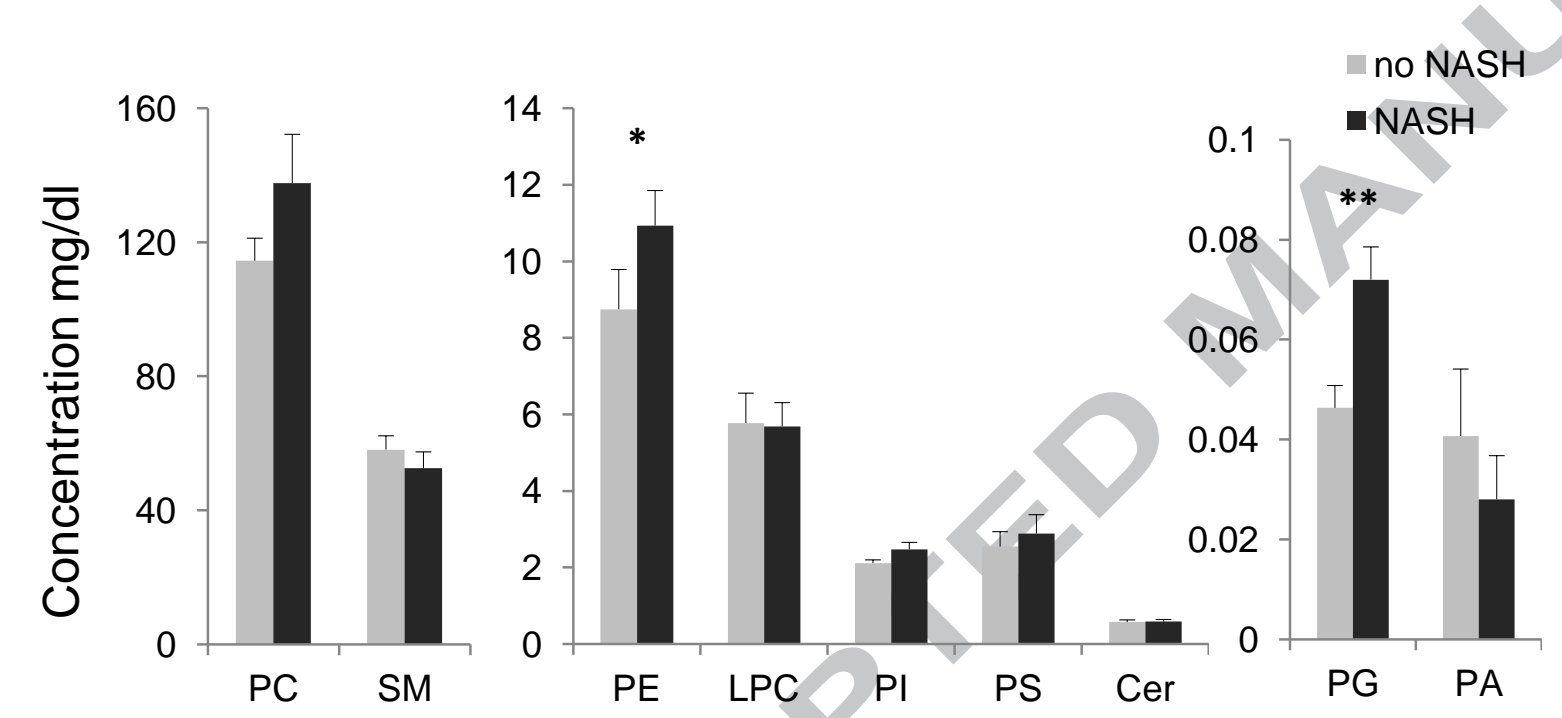

Figure 3

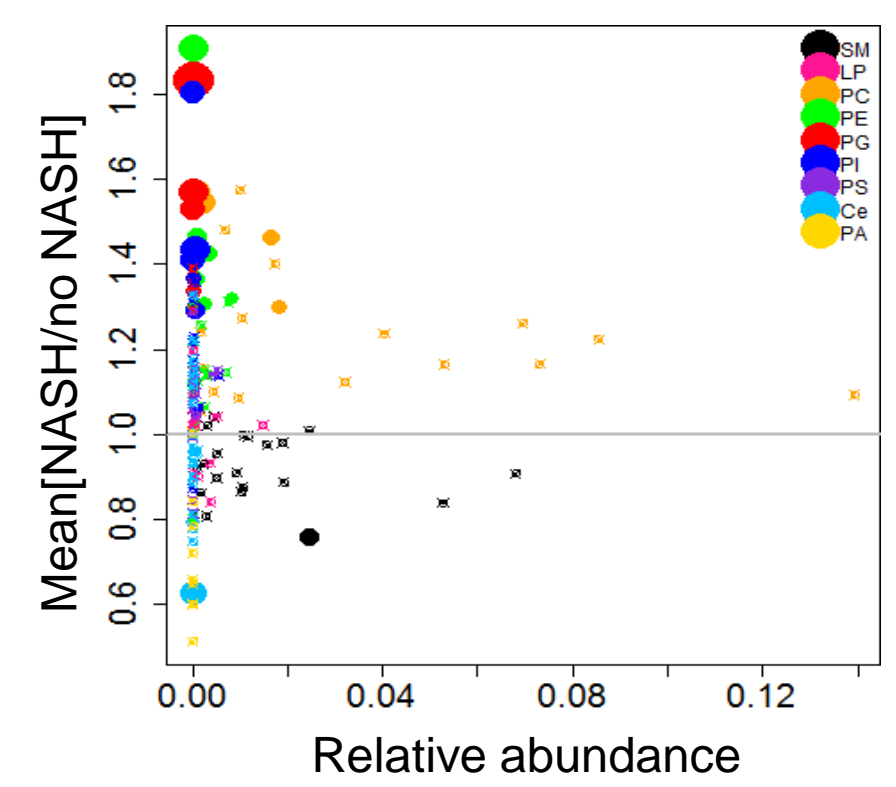


A

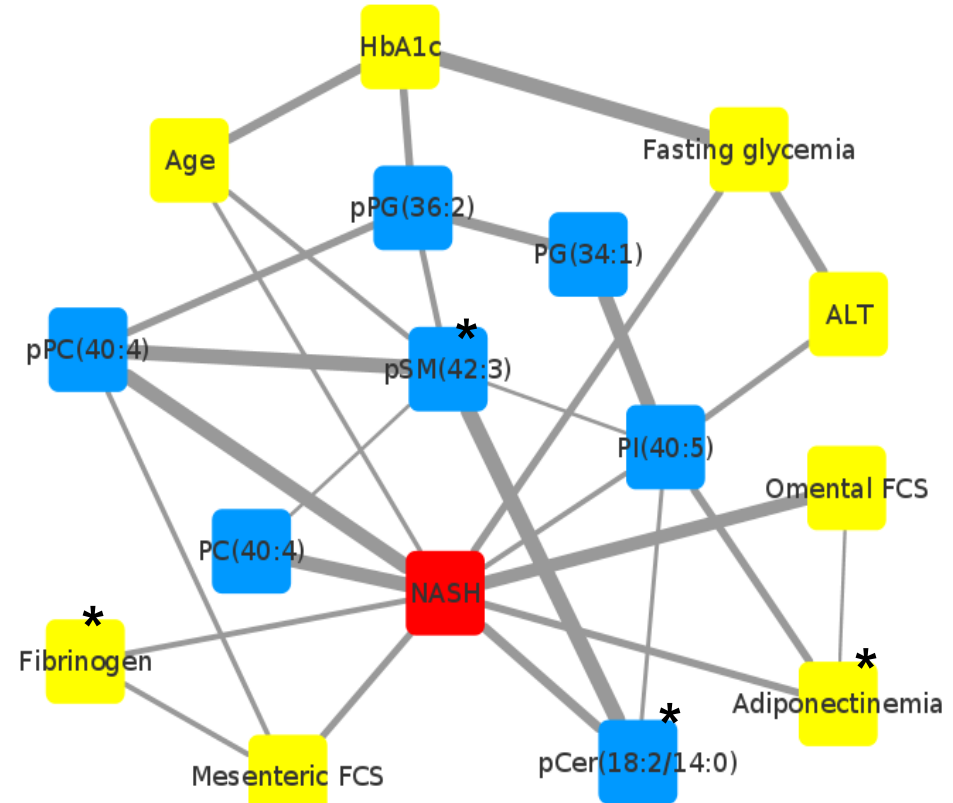

B

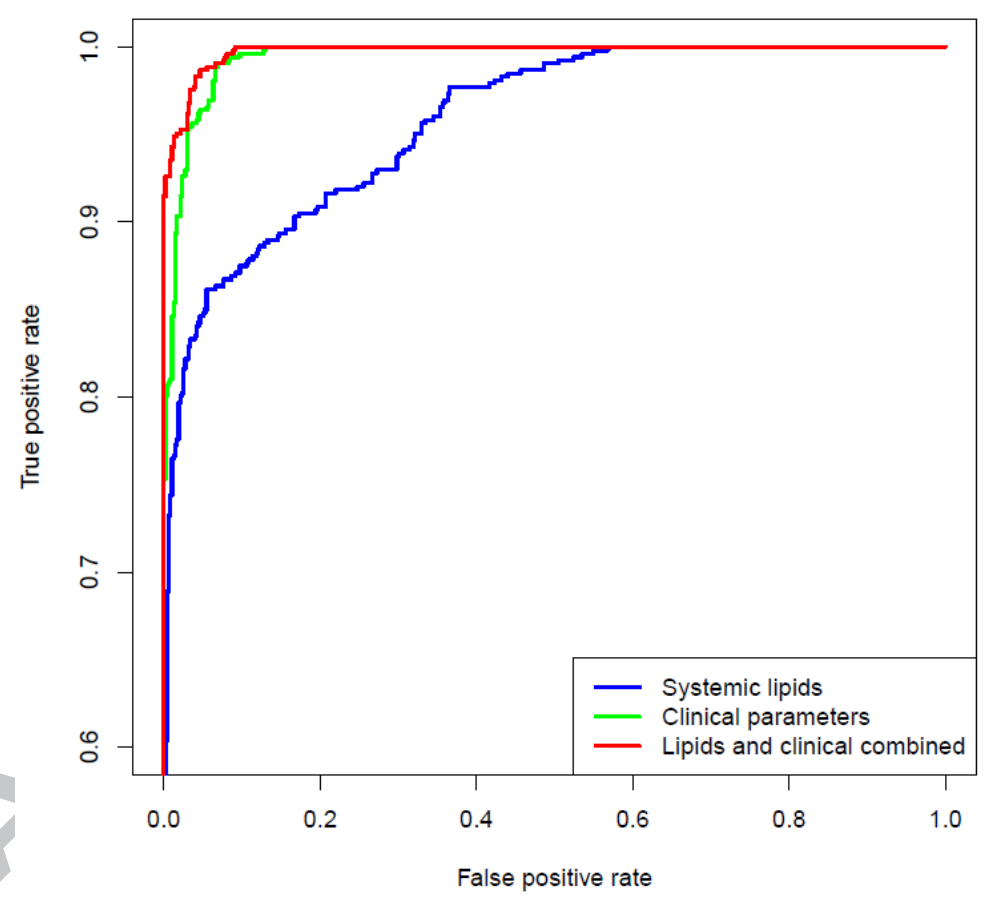

Figure 4 